\title{
Inductive Logic Programming
}

\author{
Luc De Raedt
}

Department of Computer Science, Katholieke Universiteit Leuven, Celestijnenlaan 200A, BE - 3001 Heverlee, Belgium, email: luc.deraedt@cs.kuleuven.be

\section{Definition}

Inductive logic programming is the subfield of machine learning that uses first order logic to represent hypotheses and data. Because first order logic is expressive and declarative, inductive logic programming specfically targets problems involving structured data and background knowledge. Inductive logic programming tackles a wide variety of problems in machine learning, including classification, regression, clustering, and reinforcement learning, often using "upgrades" of existing propositional machine learning systems, It relies on logic for knowledge representation and reasoning purposes. Notions of coverage, generality, and operators for traversing the space of hypotheses are grounded in logic, see also the logic of generality. Inductive logic programming systems have been applied to important applications in bio- and chemo-informatics, natural language processing, and web mining.

\section{Synonyms}

relational learning, multi-relational data mining, relational data mining, learning in logic.

\section{Motivation}

The first motivation and most important motivation for using inductive logic programming is that it overcomes the representational limitations of attribute-value learning systems. Such systems employ a table based representations where the instances correspond to rows in the table, the attributes to collumns, and for each instance, a single value is assigned to each of the attributes. This is sometimes called the single-table single tuple assumption. Many problems, such as the Bongard problem shown in Figure 1, cannot elegantly be described in this format. Bongard [1970] introduced about a 100 concept-learning or pattern recognition problems, each containing six positive and six negative examples. Even though Bongard 
problems are toy-problems, they are similar to real-life problems such as structure activity relationship prediction, where the goal is to learn to predict whether a given molecule (as represented but its 2D graph structure) are active or not. It is hard - if not, impossible - to squeeze this type of problem into the single-table single-tuple format for various reasons. Attribute-value learning systems employ a fixed number of attributes and also assume that these attributes are present in all of the examples. This assumption does not hold for the Bongard problems as the examples posses a variable number of objects (shapes). The singe-table singletuple representation imposes an implicit order on the attributes, whereas there is no natural order on the objects in the Bongard problem. Finally, the relationships between the objects in the Bongard problem are essential and must be encoded as well. It is unclear how to do this within the single-table single-tuple assumption. First order logic and relational representations allow one to encode problems involving multiple objects (or entities) as well as the relationships that hold them in a natural way.

The second motivation for using inductive logic programming is that it employs logic, a declarative representation. This implies means that hypotheses are understandable and interpretable. By using logic, inductive logic programming systems are also able to employ background knowledge in the induction process. Background knowledge can be provided in the form of definitions of auxiliary relations or predicates that may be used by the learner. Finally, logic provides a well-understood theoretical framework for knowledge representation and reasoning. This framework is also useful for machine learning, in particular for defining and developing notions such as the covers relation, generality, and refinement operators, see also the logic of generality.

\section{Theory}

Inductive logic programming is usually defined as concept-learning using logical representations. It aims at finding a hypothesis (a set of rules) that covers all positive examples and none of the negatives, while taking account a background theory. This is typically realized by searching a space of possible hypotheses. More formally, the traditional inductive logic programming definition reads as follows:

\section{Given}

- a language describing hypotheses $\mathcal{L}_{h}$,

- a language describing instances $\mathcal{L}_{i}$, 
- possibly a background theory $B$, usually in the form of a set of a (definite) clauses,

- the covers relation that specifies the relation between $\mathcal{L}_{h}$ and $\mathcal{L}_{i}$, that is when an example $e$ is covered (considered positive) by a hypothesis $h$, possibly taking into account the background theory $B$,

- a set of positive and negative examples $E=P \cup N$

Find a hypothesis $h \in \mathcal{L}_{h}$ such that for all $p \in P: \operatorname{covers}(B, h, p)=$ true and for all $n \in N: \operatorname{covers}(B, h, n)=$ false.

This definition can, as for concept-learning in general, be extended to cope with noisy data by relaxing the requirement that all examples be classified correctly.

There exist different ways to represent learning problems in logic, resulting in different learning settings. They typically use definite clause logic as the hypothesis language $\mathcal{L}_{i}$, but differ in the notion of an example. One can learn from entailment, from interpretations or from proofs, cf. the logic of generality. The most popular setting is learning from entailment, where each example is a clause and $\operatorname{covers}(B, h, e)=$ true if and only if $B \cup h \models e$.

The top leftmost scene in the Bongard problem of Figure 1 can be represented by the clause:

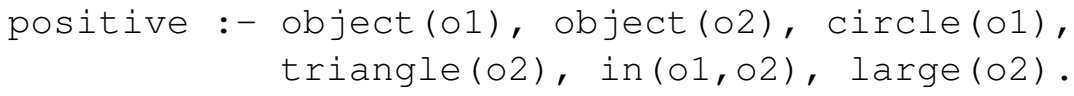

The other scenes can be encoded in the same way. The following hypothesis then forms a solution to the learning problem:

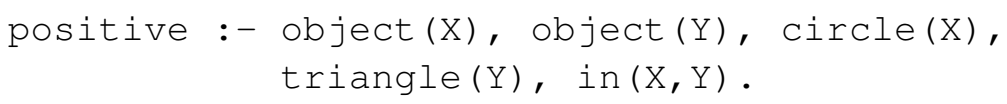

It states that those scenes having a circle inside a triangle are positive. For some more complex Bongard problems it could be useful to employ background knowledge. It could, for instance, state that triangles are polygons.

$$
\text { polygon(X) :- triangle(X). }
$$

Using this clause as background theory, an alternative hypothesis covering all positives and none of the negatives is

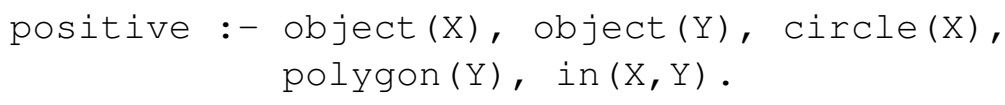




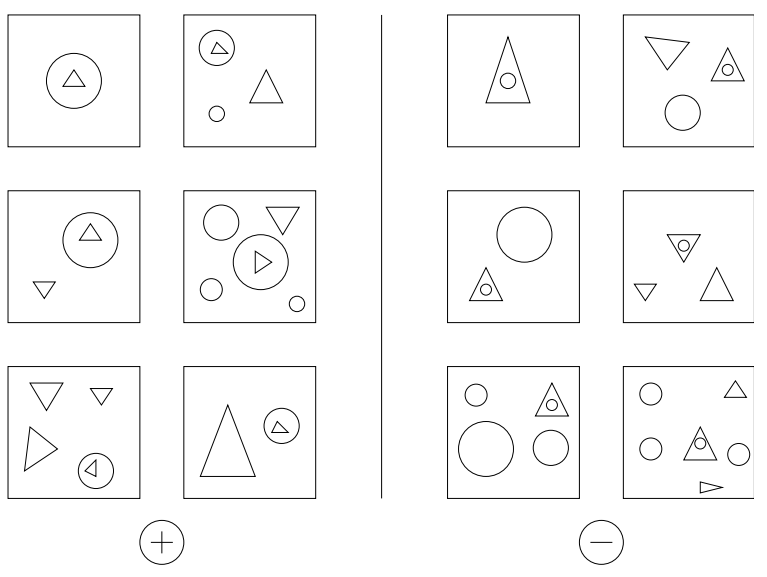

Figure 1: A complex classification problem: Bongard problem 47, developed by the Russian scientist Bongard [1970]. It consists of 12 scenes (or examples), six of class $\oplus$ and six of class $\ominus$. The goal is to discriminate between the two classes. 
An alternative for using long clauses as examples is to provide an identifier for each example and to add the corresponding facts from the condition part of the clause to the background theory. For the above exammple, the following facts

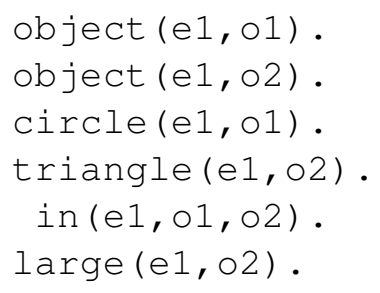

would be added to the background theory and the positive example itself would then be represented through the fact positive $(e 1)$, where e1 is the identifier. The inductive logic programming literature typically employs this format for examples and hypotheses.

Whereas inductive logic programming originally focussed on concept-learning — as did the whole field of machine learning — it is now being applied to virtually all types of machine learning problems, including regression, clustering, distancebased learning, frequent pattern mining, reinforcement learning and even kernel methods and graphical models.

\section{A Methodology}

Many of the more recenltly developed inductive logic programming systems have started from an existing attribute-value learner and have upgraded it towards the use of first order logic [Van Laer and De Raedt, 2001]. By examining state-of-the-art inductive logic programming systems one can identify a methodology for realizing this [Van Laer and De Raedt, 2001]. It starts from an attribute-value learning problem and system of interest, and takes the following two steps. First, the problem setting is upgraded by changing the representation of the examples, the hypotheses as well as the covers relation towards first order logic. This step is essentially concerned with defining the learning setting, and possible settings to be considered include the already mentioned learning from entailment, interpretations and proofs settings. Once the problem is clearly defined, one can attempt to formulate a solution. Thus the second step adapts the original algorithm to deal with the upgraded representations. While doing so, it is advisable to keep the changes as minimal as possible. This step often involves the modification of the operators used to traverse the search space. Different operators for realizing this are introduced in the entry on the logic of generality'.

There are many reasons why following the methodology is advantageous. First, by upgrading a learner that is already effective for attribute-value representations, 
one can benefit from the experiences and results obtained in the propositional setting. In many cases, for instance decision trees, this implies that one can rely on well-established methods and findings, which are the outcomes of several decades of machine learning research. It will be hard to do better starting from scratch. Second, upgrading an existing learner is also easier than starting from scratch as many of the components (such as heuristics and search strategy), can be recycled. It is therefore also economic in terms of man power. Third, the upgraded system will be able to emulate the original one, which provides guarantees that the output hypotheses will perform well on attribute value learning problems. Even more important is that it will often also be able to emulate extensions of the original systems. For instance, many systems that extend frequent item-set mining towards using richer representations, such as sequences, intervals, the use of taxonomies, graphs, ..., have been developed over the past decade. Many of them can be emulated using the inductive logic programming upgrade of Apriori [Agrawal et al., 1996] called Warmr [Dehaspe and Toivonen, 2001]. The upgraded inductive logic programming systems will typically be more flexible than the systems it can emulate but typically also less efficient because there is a price to be paid for expressiveness. Finally, it may be possible to incorporate new features in the attribute value learner by following the methodology. One feature that is often absent from propositional learners and may be easy to incorporate, is the use of a background theory.

It should be mentioned that the methodology is not universal, that is, there exist also approaches, such as Muggleton's Progol [Muggleton, 1995], which have directly been developed in first order logic and for which no propositional counter part exists. In such cases, however, it can be interesting to follow the inverse methodology, which would specialize the inductive logic programming system.

\section{FOIL: an illustration}

One of the simplest and best-known inductive logic programming systems is FOIL [Quinlan, 1990]. It can be regarded as an upgrade of a rule-learner such as CN2 [Clark and Niblett, 1989]. FOIL's problem-setting is an instance of the learning from entailment setting introduced above (though it restricts the background theory to ground facts only and does not allow functors).

Like most rule-learning systems, FOIL employs a separate-and-conquer approach. It starts from the empty hypothesis, and then repeatedly searches for one rule that covers as many positive examples as possible and no negative example, adds it to the hypothesis, removes the positives covered by the rule, and then iterates. This process is continued untill all positives are covered. To find one rule, 
it performs a hill-climbing search through the space of clauses ordered according generality. The search starts at the most general rule, the one stating that all examples are positive, and then repeatedly specializes it. Among the specializations it then selects the best one according to a heuristic evaluation based on information gain. A heuristic, based on the minimum description length principle, is then used to decide when to stop specializing clauses.

The key differences between FOIL and its propositional pre-decessors are the representation and the operators used to compute the specializations of a clause. It it employs a refinement operator under $\theta$-subsumption [Plotkin, 1970] (see also the logic of generality). Such an operator essentially refines clauses by adding atoms to the condition part of the clause or applying substitutions to a clause. For instance, the clause

positive :- triangle $(X)$, in $(X, Y), \operatorname{color}(X, C)$.

can be specialized to

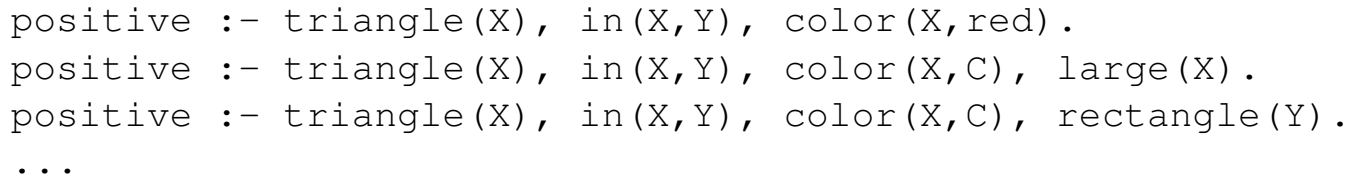

The first specialization is obtained by substituting the variable $C$ by the constant red, the other two by adding an atom (large (X), resp. rectangle (Y)) to the condition part of the rule. Inductive logic programming systems typically also employ syntactic restrictions - the so-called bias — that specify which clauses may be used in hypotheses. For instance, in the above example, the second argument of the color predicate belongs to the type Color, whereas the arguments of in are of type Object and consists of object identifiers.

\section{Application}

Inductive logic programming has been succesfully applied to many application domains, including: bio- and chemo-informatics, ecology, network mining, software engineering, information retrieval, music analysis, webmining, natural language processing, toxicology, robotics, program synthesis, design, architecture, and many others. The best-known applications are in scientific domains. For instance, in structure activity relationship prediction, one is given a set of molecules together with their activities, and background knowledge encoding functional groups, that is particular components of the molecule, and the task is to learn rules stating when 


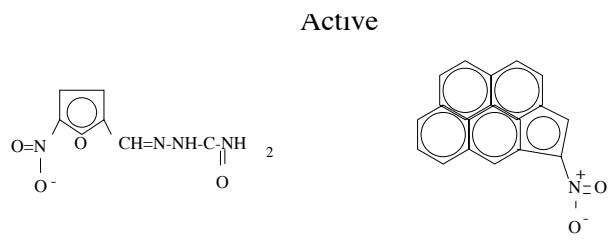

nitrofurazone 4-nitropenta[cd]pyrene

Structural alert:

Inactive
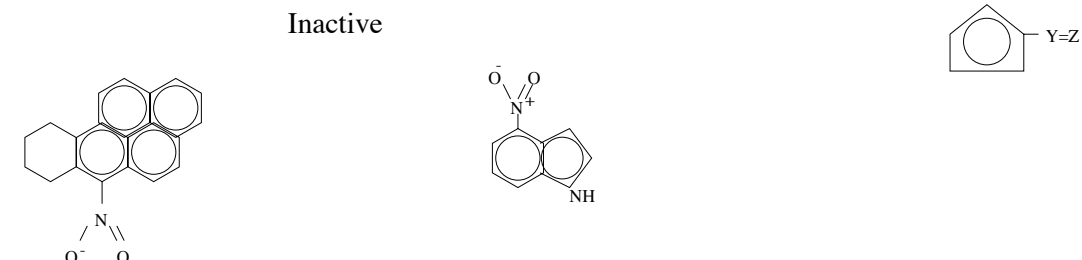

6-nitro-7,8,9,10-tetrahydrobenzo[a]pyrene

4-nitroindole

Figure 2: Predicting mutagenicity, cf. Srinivasan et al. [1996].

a molecule is active or inactive. This is illustrated in Figure 2 (after Srinivasan et al. [1996]), where two molecules are active and two are inactive. One then has to find a pattern that discriminates the actives from the inactives. Structure activity relationship prediction (SAR) is an essential step in for instance drug discovery. Using the general purpose inductive logic programming system Progol [Muggleton, 1995] structural alerts, such as that shown in Figure 2, have been discovered. These alerts allow one to distinguish the actives from the inactives - the one shown in the figure matches both of hte actives but none of the inactives - and at the same time they are readily interpretable and provide useful insight into the factors determining the activity. To solve structural activity relationship prediction problems using inductive logic programming one must represent the molecules and hypotheses using the logical formalisms introduced above. The resulting representation is very simlar to that employed in the Bongard problems: the objects are the atoms and relationships the bonds. Particular functional groups are encoded as background predicates. 


\section{State-of-the-art}

The upgrading methodology has been applied to a wide-variety of machine learning systems and problems. There exist now inductive logic programming systems that

- induce logic programs from examples under various learning settings. This is by far the most popular class of inductive logic programming systems. Well-known system include Aleph [Srinivasan, 2007] and Progol [Muggleton, 1995] as well as various variants of FOIL [Quinlan, 1990]. Some of these systems, especially Progol and Aleph contain many features that are not present in propositional learning systems. Most of these systems focus on a classification setting, and learn the definition of a single predicate.

- induce logical decision trees from examples. These are binary decision trees containing conjunctions of atoms (that is queries) as tests. If a query succeeds, then one branch is taken, otherwise the other one. Decision trees methods for both classification and regression exist, see [Blockeel and De Raedt, 1998] and [Kramer and Widmer, 2001].

- mine for frequent queries, where queries are conjunctions of atoms. Such queries can be evaluated on an example. For instance, in the Bongard problem, the query ?- triangle $(X)$, in $(X, Y)$ succeeds on the leftmost scenes, and fails on the rightmost ones. Therefore, its frequency would be 6 . The goal is then to find all queries that are frequent, that is whose frequencie exceeds a certain threshold. Frequent query mining ugrades the popular local pattern mining setting due to Agrawal et al. [1996] to inductive logic programming, see [Dehaspe and Toivonen, 2001].

- learn or revise the definitions of theories, which consist of the definitions of multiple predicates, at the same time, cf. [Wrobel, 1996] and the entry Theory revision in this encyclopedia. Several of these systems have their origin in the Model Inference System by Shapiro [1983] or the work by Angluin [1987].

\section{Current Trends and Challenges}

There are two major trends and chanllenges in inductive logic programming. The first challenge is to extend the inductive logic programming paradigm beyond the purely symbolic one. Important trends in this regards include 
- the combination of inductive logic programming principles with graphical and probabilistic models for reasoning about uncertainty. This is a field known as statistical relational learning, probabilistic logic learning or probabilistic inductive logic programming. At the time of writing this is a very popular research stream, attracting a lot of attention in the wider artificial intelligence community, cf. the entry Statistical Relational Learning in this encyclopedia. It has resulted in many relational or logical upgrades of wellknown graphical models including bayesian networks, Markov networks, hidden Markov models, and stochastic grammars.

- the use of relational distance measures for classification and clustering Ramon and Bruynooghe [1998], Kirsten et al. [2001]. These distances measure the similarity between two examples or clauses, while taking into account the underlying structure of the instances. These distances are then combined with standard classification and clustering methods such as $k$-nearest neighbor and $k$-means.

- the integration of relational or logical representations in reinforcement learning, known as relational reinforcement learning [Džeroski et al., 2001].

The power of inductive logic programming is also its weakness. The ability to represent complex objects and relations and the ability to make use of background knowledge add to the computational complexity. Therefore, a a key challenge of inductive logic programming is tackling this added computational complexity. Even the simplest method for testing whether one hypothesis is more general than another - that is $\theta$-subsumption [Plotkin, 1970] — is NP-complete. Similar tests are used for deciding whether a clause covers a particular example in systems such as FOIL. Therefore inductive logic programming and relational learning systems are computationally much more expensive than their propositional counter parts. This is an instance of the expressiveness versus efficiency trade-off in computer science. Because of these computational difficulties, inductive logic programming has devoted a lot of attention efficiency issues. On the theoretical side, there exist various results about the polynomial learnability of certain subclasses of logic programs, cf. [Cohen and Page, 1995] for an overview. From a practical perspective, there is quite some work on developing efficient methods for searching the hypothesis space and especially for evaluating the quality of hypotheses. Many of these methods employ optimized inference engines based on Prolog or database technology or constraint-satisfaction methods, cf. Blockeel and Sebag [2003] for an overview. 


\section{Further Reading}

A comprehensive introduction to inductive logic programming can be found in the book by De Raedt [2008] on logical and relational learning. Early surveys of inductive logic programming are contained in [Muggleton and De Raedt, 1994] and [Lavrač and Džeroski, 1994] and an account of its early history is provided in [Sammut, 1993]. More recent collections on current trends can be found in the proceedings of the annual Inductive Logic Programming Conference (publishes in Springer's Lectures Notes in Computer Science Series) and special issues of the Machine Learning Journal. An interesting collection of inductive logic programming and multi-relational data mining work is provided in [Džeroski and Lavrač, 2001]. The upgrading methodology is described in detail in [Van Laer and De Raedt, 2001]. More information on logical issues in inductive logic programming are given in the entry The logic of generality in this encyclopedia, whereas the entries statistical relational learning and graph mining are recommended for those interested in frameworks tackling similar problems using other types of representations.

\section{References}

R. Agrawal, H. Mannila, R. Srikant, H. Toivonen, and A.I. Verkamo. Fast discovery of association rules. In U. Fayyad, G. Piatetsky-Shapiro, P. Smyth, and R. Uthurusamy, editors, Advances in Knowledge Discovery and Data Mining, pages 307-328. MIT Press, 1996.

D. Angluin. Queries and concept-learning. Machine Learning, 2:319-342, 1987.

H. Blockeel and L. De Raedt. Top-down induction of first order logical decision trees. Artificial Intelligence, 101(1-2):285-297, 1998.

H. Blockeel and M. Sebag. Scalability and efficiency in multi-relational data mining. SIGKDD Explorations, 5(1):17-30, 2003.

M. Bongard. Pattern Recognition. Spartan Books, 1970.

P. Clark and T. Niblett. The CN2 algorithm. Machine Learning, 3(4):261-284, 1989.

W. W. Cohen and D. Page. Polynomial learnability and inductive logic programming: Methods and results. New Generation Computing, 13, 1995.

Luc De Raedt. Logical and Relational Learning. Springer, 2008. To appear. 
L. Dehaspe and H. Toivonen. Discovery of relational association rules. In S. Džeroski and N. Lavrač, editors, Relational Data Mining, pages 189-212. Springer, 2001.

S. Džeroski, L. De Raedt, and K. Driessens. Relational reinforcement learning. Machine Learning, 43(1/2):5-52, 2001.

S. Džeroski and N. Lavrač, editors. Relational Data Mining. Springer, 2001.

M. Kirsten, S. Wrobel, and T. Horvath. Distance based approaches to relational learning and clustering. In S. Džeroski and N. Lavrač, editors, Relational Data Mining, pages 213-232. Springer, 2001.

Stefan Kramer and Gerhard Widmer. Inducing classification and regression trees in first order logic. In S. Džeroski and N. Lavrač, editors, Relational Data Mining, pages 140-159. Springer, 2001.

N. Lavrač and S. Džeroski. Inductive Logic Programming: Techniques and Applications. Ellis Horwood, 1994.

S. Muggleton. Inverse entailment and Progol. New Generation Computing, 13, 1995.

S. Muggleton and L. De Raedt. Inductive logic programming: Theory and methods. Journal of Logic Programming, 19/20:629-679, 1994.

G. D. Plotkin. A note on inductive generalization. In Machine Intelligence, volume 5, pages 153-163. Edinburgh University Press, 1970.

J. R. Quinlan. Learning logical definitions from relations. Machine Learning, 5: 239-266, 1990.

J. Ramon and M. Bruynooghe. A framework for defining distances between firstorder logic objects. In D. Page, editor, Proceedings of the 8th International Conference on Inductive Logic Programming, volume 1446 of Lecture Notes in Artificial Intelligence, pages 271-280. Springer, 1998.

C. Sammut. The origins of inductive logic programming: A prehistoric tale. In S. Muggleton, editor, Proceedings of the 3rd International Workshop on Inductive Logic Programming, pages 127-148. J. Stefan Institute, 1993.

E. Y. Shapiro. Algorithmic Program Debugging. MIT Press, 1983.

A. Srinivasan. The Aleph Manual, 2007. URL http://www2 .comlab.ox . ac.uk/oucl/research/areas/machlearn/Aleph/aleph_toc.html. 
A. Srinivasan, S. Muggleton, M. J. E. Sternberg, and R. D. King. Theories for mutagenicity: A study in first-order and feature-based induction. Artificial Intelligence, 85(1/2):277-299, 1996.

W. Van Laer and L. De Raedt. How to upgrade propositional learners to first order logic: A case study. In S. Džeroski and N. Lavrač, editors, Relational Data Mining, pages 235-261. Springer, 2001.

S. Wrobel. First-order theory refinement. In L. De Raedt, editor, Advances in Inductive Logic Programming, volume 32 of Frontiers in Artificial Intelligence and Applications, pages 14-33. IOS Press, 1996. 